\title{
Potential of chloroquine and hydroxychloroquine to treat COVID-19 causes fears of shortages among people with systemic lupus erythematosus
}

To the Editor - In March 2020, in the midst of the COVID-19 pandemic ${ }^{1}$, reports that chloroquine (CQ) can inhibit the growth of severe acute respiratory syndrome coronavirus (SARS-CoV-2) ) $^{2,3}$ have brought this molecule into the spotlight. Hydroxychloroquine (HCQS), which is typically used in dermatology clinics for the management of systemic lupus erythematosus (SLE) and has a better clinical safety profile and fewer drug-drug interactions than CQ, has also been demonstrated to have anti-SARS$\mathrm{CoV}$ activity in vitro ${ }^{2,4}$. Now, amid the speculation regarding the beneficial roles of these molecules in COVID-19 treatment, shortages of CQ and HCQS are feared.

CQ has been shown in vitro to increase endosomal $\mathrm{pH}$, prevent virus-cell fusion, and interfere with glycosylation of the ACE2 receptor and thus the binding of the SARSCoV-2 $\mathrm{S}$ protein to ACE2 (ref. ${ }^{3}$ ). Chinese experts recommend that mild, moderate and severe COVID-19 cases (without contraindications to $\mathrm{CQ}$ ) be treated with 500 milligrams of CQ twice daily for 10 days (ref. ${ }^{5}$ ). A Chinese study has demonstrated that HCQS is more potent than CQ in inhibiting SARS-CoV-2 in vitro ${ }^{2}$. These new developments have brought some hope for COVID-19 treatment, though more research is warranted to validate the findings.

A shortage in HCQS would create problems for people with SLE who are currently taking this drug. Given the reports of the effectiveness of HCQS in COVID-19 treatment and the anticipated shortage of HCQS, the number of patients with SLE visiting dermatology outpatient departments (even before their scheduled appointments) to refill their medication has increased. SLE is a chronic autoimmune disease with varied manifestations (including fever, rash, alopecia, arthralgia, fatigue and photosensitivity), which usually follows a relapsing and remitting course. Various organs, such as the skin, kidneys, heart, joints, pleura, pericardium and brain, may be involved in the disease process. The use of HCQS for SLE offers several advantages by controlling constitutional symptoms; decreasing the risk of flare-ups; sparing the use of glucocorticosteroids; decreasing the risk of organ damage, osteoporosis and thromboembolism; and increasing life expectancy ${ }^{6}$. In addition, HCQS is considered safe in pregnancy, and it has a protective role in preventing congenital heart block. A prolonged shortage of the drug might deprive people with SLE of a low-cost, safe, effective and well-tolerated drug. Patients are terrified that they might lose access to the drug that prevents their disease from damaging other vital organs.

Beyond affecting people with SLE, the shortage of CQ and HCQS would affect people with other rheumatological disorders, such as rheumatoid arthritis, primary Sjögren syndrome and antiphospholipid syndrome ${ }^{6}$. In addition, HCQS is commonly prescribed for various dermatological indications including dermatomyositis, sarcoidosis, polymorphous light eruptions, chronic actinic dermatitis, granuloma annulare, lichen planus, chronic erythema nodosum and morphea. Patients taking chloroquine analogs for its metabolic, cardiovascular, antithrombotic and antineoplastic effects may also be negatively affected by a shortage.

The pharmaceutical companies and other stakeholders manufacturing HCQS should quickly assess the situation and make sure that the drug remains available to those who need it. In addition, people should not purchase medications that they do not need, and health authorities should make sure that HCQS is not dispensed without a prescription. Combined sensible efforts can help us navigate these difficult times.

Deepak Jakhar (D) $₫$ and Ishmeet Kaur Department of Dermatology, North Delhi Municipal Corporation Medical College and Hindu Rao Hospital, New Delhi, India.

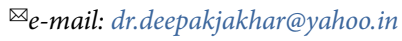

Published online: 8 April 2020

https://doi.org/10.1038/s41591-020-0853-0

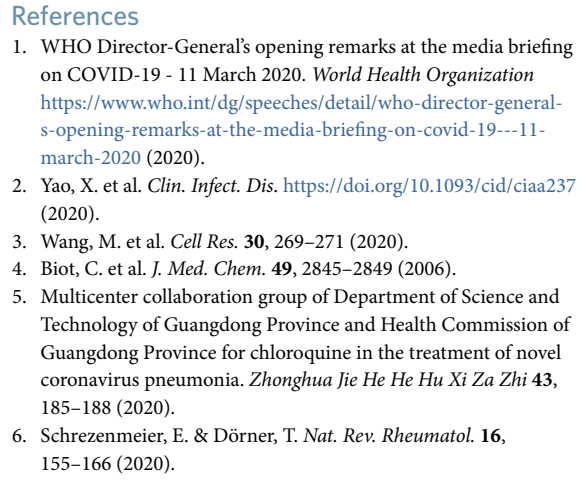

1. WHO Director-General's opening remarks at the media briefing on COVID-19 - 11 March 2020. World Health Organization https://www.who.int/dg/speeches/detail/who-director-generals-opening-remarks-at-the-media-briefing-on-covid-19---11march-2020 (2020).

2. Yao, X. et al. Clin. Infect. Dis. https://doi.org/10.1093/cid/ciaa237 (2020).

3. Wang, M. et al. Cell Res. 30, 269-271 (2020)

4. Biot, C. et al. J. Med. Chem. 49, 2845-2849 (2006).

5. Multicenter collaboration group of Department of Science and Technology of Guangdong Province and Health Commission of Guangdong Province for chloroquine in the treatment of novel coronavirus pneumonia. Zhonghua Jie He He Hu Xi Za Zhi 43, 185-188 (2020).

6. Schrezenmeier, E. \& Dörner, T. Nat. Rev. Rheumatol. 16, 155-166 (2020).

\section{Coping with COVID-19: scaling up virtual care to standard practice}

To the Editor - In times of disease outbreak, social distancing can be facilitated by video consultation, but many practices are not ready to implement this. Here, we share a roadmap for emergency scaling up of virtual care in the outpatient setting.
Since the outbreak of SARS CoV-2

and the associated disease, COVID-19, in December of 2019, the effects of the virus 